\title{
Results of a Gene Panel Approach in a Cohort of Patients with Incomplete Distal Renal Tubular Acidosis and Nephrolithiasis
}

\author{
Viola D'Ambrosio a, b Alessia Azzaràc Eugenio Sangiorgib, c Fiorella Gurrierid \\ Bernhard Hess $^{e}$ Giovanni Gambaro ${ }^{f}$ Pietro Manuel Ferraro ${ }^{a, b}$ \\ aU.O.S. Terapia Conservativa della Malattia Renale Cronica, U.O.C. Nefrologia, Fondazione Policlinico Universitario

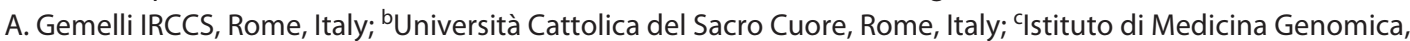 \\ Fondazione Policlinico Universitario A. Gemelli IRCCS - Università Cattolica del Sacro Cuore, Rome, Italy; \\ d Unità di genetica Medica e Funzionale, Università Campus Bio-Medico, Rome, Italy; eInternal Medicine and \\ Nephrology, Klinik Im Park, Zurich, and University of Zurich, Zurich, Switzerland; ${ }^{\mathrm{f}}$ Renal Unit, Division of Nephrology \\ and Dialysis, Department of Medicine, University of Verona, Verona, Italy
}

\section{Keywords}

Nephrolithiasis · Nephrocalcinosis · Genetics · Distal renal tubular acidosis · Metabolic acidosis

\begin{abstract}
Background: Distal renal tubular acidosis (dRTA) is characterized by an impairment of urinary acidification resulting in metabolic acidosis, hypokalemia, and inappropriately elevated urine $\mathrm{pH}$. If not treated, this chronic condition eventually leads to nephrocalcinosis, nephrolithiasis, impaired renal function, and bone demineralization. dRTA is a well-defined entity that can be diagnosed by genetic testing of 5 genes known to be disease-causative. Incomplete dRTA (idRTA) is defined as impaired urinary acidification that does not lead to overt metabolic acidosis and therefore can be diagnosed if patients fail to adequately acidify urine after an ammonium chloride $\left(\mathrm{NH}_{4} \mathrm{Cl}\right)$ challenge or furosemide and fludrocortisone test. It is still uncertain whether idRTA represents a distinct entity or is part of the dRTA spectrum and whether it is caused by mutations in the same genes of overt dRTA. Methods: In this cross-sectional study, we investigated a group of 22 stone formers whose clinical features were suspicious of idRTA. They underwent an $\mathrm{NH}_{4} \mathrm{Cl}$ challenge
\end{abstract}

karger@karger.com www.karger.com/kbr

Karger"
(C) 2021 The Author(s)

Published by S. Karger AG, Basel

This is an Open Access article licensed under the Creative Commons Attribution-NonCommercial-4.0 International License (CC BY-NC) (http://www.karger.com/Services/OpenAccessLicense), applicable to the online version of the article only. Usage and distribution for commercial purposes requires written permission. and were found to have impaired urinary acidification ability. These patients were then analyzed by genetic testing with sequencing of 5 genes: SLC4A1, ATP6V1B1, ATP6VOA4, FOXI1, and WDR72. Results: Two unrelated individuals were found to have two different variants in SLC4A1 that had never been described before. Conclusions: Our results suggest the involvement of other genes or nongenetic tubular dysfunction in the pathogenesis of idRTA in stone formers. However, genetic testing may represent a cost-effective tool to recognize, treat, and prevent complications in these patients.

(c) 2021 The Author(s)

Published by S. Karger AG, Basel

\section{Introduction}

One of the main functions of the kidney is to maintain the acid-base balance. This task is carried out by tubules that are responsible for urinary acidification and elimination of nonvolatile acids. When the kidney is no longer able to eliminate acidic metabolism waste products, a normal serum anion gap or hyperchloremic metabolic acidosis can develop; this condition is called renal tubular acidosis (RTA). 
Table 1. Secondary forms of dRTA

Sjögren syndrome and other autoimmune diseases

Kidney transplantation

Medullary sponge kidney

Chronic obstructive uropathy

Drugs (amphotericin B, foscarnet, and lithium)

Cirrhosis

Sickle cell anemia

dRTA, distal renal tubular acidosis.

Of the 4 types of RTA [1], type 1 or distal RTA (dRTA) is characterized by an impairment of urinary acidification resulting in a hyperchloremic nonanion gap metabolic acidosis, hypokalemia, and inappropriately elevated urine $\mathrm{pH}[2,3]$. If not treated, this chronic condition eventually leads to nephrocalcinosis and recurrent nephrolithiasis, impaired renal function, and bone demineralization due to reabsorption of bicarbonate and phosphate complexed with calcium from the bone as a buffer for metabolic acidosis [4]. Acquired dRTA may result from different kinds of tubulointerstitial damage (Table 1) [5]. Hereditary dRTA can be diagnosed by genetic testing of 5 genes known to be disease-causative (ATP6V1B1, ATP6V0A4, FOXI1, SLC4A1, and WDR72) [6].

Urinary acidification takes place primarily in the alpha intercalated cells (alpha-ICs) of the collecting duct, which are responsible for $\mathrm{H}^{+}$and $\mathrm{HCO}_{3}{ }^{-}$secretion. In the luminal membrane of alpha-ICs, vacuolar $\mathrm{H}^{+}$-ATPase and $\mathrm{H}^{+} / \mathrm{K}^{+}$-ATPase are involved in luminal $\mathrm{H}^{+}$secretion. At the same time, intracellular $\mathrm{HCO}_{3}{ }^{-}$formed by the cytosolic carbonic anhydrase II leaves the cell by a $\mathrm{Cl}^{-} / \mathrm{HCO}_{3}{ }^{-}$ anion exchanger (AE1 or band 3 protein) located in the basolateral membrane [7], the exchange of $\mathrm{Cl}^{-}$for $\mathrm{HCO}_{3}{ }^{-}$ allows the reabsorption of bases, but most importantly plays a crucial role in urinary acidification. However, pathogenic variants in genes coding for the aforementioned transporters have been identified as causes of inherited (primary) dRTA resulting in secretory defects. Genetic dRTA can be transmitted in an autosomal dominant or recessive manner depending on the gene involved. Pathogenic variants in ATP6V0A4, ATP6V1B1, FOXI1, and WDR72 are associated with autosomal recessive inheritance, while pathogenic variants in SLC4A1 are associated with both autosomal dominant and recessive inheritance [8].

ATP6V0A4 (7q34) and ATP6V1B1 (2p13.3) encode for the alpha-4 subunit and the beta- 1 subunit of the vacuolar $\mathrm{H}^{+}$-ATPase [9]. FOXI1 (5q35.1) encodes for a tran- scription factor (forkhead box protein I1) that regulates the function of AE1 and AE4 and V-ATPase subunits. SLC4A1 (17q21.31) encodes for AE1 located on the basolateral membrane of alpha-IC. Both dominant and recessive SLC4A1 variants have been described [9-11]. The most recent gene to be identified as a cause of dRTA is WDR72 (15q21.3). This gene encodes for an intracellular protein whose function is still not very clear. Although other genes may be involved in distal tubular acidification [12], currently, these 5 genes are the only ones whose defects are known to be disease causative in humans.

The term "incomplete distal renal tubular acidosis" (idRTA) was first coined in 1959 by Wrong and Davies [13] referring to patients who could not maximally lower urine $\mathrm{pH}$ after an ammonium chloride $\left[\mathrm{NH}_{4} \mathrm{Cl}\right]$ loading test and yet did not present with overt metabolic acidosis [13-16]. idRTA is characterized by a similar but less severe clinical phenotype than overt dRTA and is defined as an impaired urinary acidification that does not lead to overt metabolic acidosis. Since the clinical features are not well-defined, it can be diagnosed if, after an $\mathrm{NH}_{4} \mathrm{Cl}$ challenge or other acid load tests, patients fail to adequately acidify their urine [15-18]. Whether this condition is a distinct clinical entity, a part of the dRTA spectrum or simply a variant of the ability to acidify urine was and still is uncertain. Although quite rare, cases of conversion from incomplete to complete dRTA have been reported proving the existence of a pathophysiological continuum between the two entities [9]. Variants in the same genes known to be disease-causative for dRTA have been described (Table 2). Heterozygous truncating mutations and subunit polymorphisms of ATP 6 V1B1 have been linked to idRTA in patients who presented with normal blood $\mathrm{pH}$, urinary acidification defect, and elevated stone risk [19, 20]. In a recent case report, Imai et al. [21] described an adult patient who was found to have an incomplete and late-onset form of dRTA and whose genetic testing was positive for a mutation of ATP6VOA4. Heterozygous ATP6V1B1 and ATP6V0A4 pathogenic variants have been linked to idRTA, as well as mutations in SLCAA1.

Incomplete dRTA has been reported to be frequent in "primary" osteoporosis, particularly in men [22, 23], and in calcium nephrolithiasis. In addition, up to $13 \%$ of recurrent "idiopathic" calcium stone formers have idRTA $[16,18,24,25]$.

So far, no studies have systematically investigated the prevalence of variants in genes known to cause dRTA in a cohort of patients diagnosed with idRTA. In fact, the majority of the previous studies were case series or case 
Table 2. Variants in the genes known to be disease-causative for dRTA associated with idRTA

\begin{tabular}{llll}
\hline Gene & $\begin{array}{l}\text { Chromosome } \\
\text { locus }\end{array}$ & Protein & Mutation \\
ATP6V0A4 & $7 \mathrm{q} 34$ & V type $\mathrm{H}^{+}$-ATPase alpha 4 subunit & Heterozygous p.S544L [21] \\
ATP6V1B1 & 2p13.3 & V type H $\mathrm{H}^{+}$-ATPase beta 1 subunit & Heterozygous truncation mutation p.F468fsX487 [27] \\
ATP6V1B1 & 2p13.3 & V type H $\mathrm{H}^{+}$-ATPase beta 1 subunit & Heterozygous nonsynonymous polymorphism p.E161K [20] \\
SLC4A1 & $17 q 21.31$ & AE1 & PRIBRAM mutation [11] \\
SLC4A1 & 17q21.31 & AE1 & Erythroid intron 3 (promoter region) mutation rs999716 [30]
\end{tabular}

dRTA, distal renal tubular acidosis; idRTA, incomplete distal renal tubular acidosis; AE1, anion exchanger 1.

Table 3. Demographic and clinical characteristics of patients who tested positive to the simplified 1-day $\mathrm{NH}_{4} \mathrm{Cl}$ challenge

\begin{tabular}{lll}
\hline & $N$ (out of 22 patients) & Prevalence (\%) \\
\hline Nephrocalcinosis & 7 & 32 \\
Hypercalciuria & 6 & 27 \\
Hyperoxaluria & 7 & 32 \\
Hypocitraturia & 9 & 41 \\
DXA & & 45 \\
$\quad$ Reduced bone density (osteoporosis or osteopenia) & 10 & 5 \\
$\quad$ Normal bone density & 1 & 50 \\
$\quad$ No DXA available & 11 & 68 \\
Gender & & 32 \\
$\quad$ Male & 15 & 91 \\
$\quad$ Female & 7 & SD \\
Recurrent stone formers (>1 episode) & 20 & 15.3 \\
\hline & Mean & 20 \\
\hline Age, years & 50.8 & 17.2 \\
Height, cm & 169 & 4.2 \\
Weight, kg & 70.8 & \\
\hline DXI, kg/m & 24.6 & \\
\hline
\end{tabular}

reports, from which an estimate of prevalence could not be determined. This study has been performed to investigate whether gene mutations in the 5 aforementioned genes are also causing the frequently observed idRTA in nephrolithiasis, using a gene panel approach.

\section{Materials and Methods}

\section{Patients}

In this cross-sectional study, we enrolled the calcium stone formers followed at the Klinik Im Park stone clinic in Zurich who tested positive to an acidification test, had a definite family history of nephrolithiasis during the period from 2012 to 2017 and in whom secondary forms of RTA were ruled out (see online suppl. Table 1; see www.karger.com/doi/10.1159/000516389 for all online suppl. material). All were of Caucasian descent. Ele- ments of suspicion to undergo the test were a history of nephrolithiasis or nephrocalcinosis with morning urinary $\mathrm{pH}>5.8$ in the absence of metabolic acidosis. To establish the diagnosis, these patients underwent a simplified 1-day $\mathrm{NH}_{4} \mathrm{Cl}$ challenge as previously described [23]. The test consists in the oral ingestion of $\mathrm{NH}_{4} \mathrm{Cl}$ at a dose of $50 \mathrm{mg} / \mathrm{kg}$ body weight in 3 doses before main meals given as $400 \mathrm{mg}$ capsules, over $1 \mathrm{~h}$ and with water to attenuate gastric irritation. After $\geq 12$ h overnight fast, the second morning urine is then collected. The diagnostic success of a test is bound to its power in inducing systemic acidosis to investigate whether this is coped by urine acidification in the distal nephron. In the series of patients described by Sromicki and Hess [23], this test was able to induce an acidemic response (within normal limits) in all patients and controls as shown not only by decreases in venous $\mathrm{pH}$ and bicarbonate but also by the drop in urinary citrate, an important marker of renal intracellular acidosis [26]. The test is considered positive (e.g., the patient affected with idRTA) if urine $\mathrm{pH}$ measured with a $\mathrm{pH}$ me- 
ter after the $\mathrm{NH}_{4} \mathrm{Cl}$ load fails to drop below 5.45 or if serum bicarbonate drops below $20.5 \mathrm{mmol} / \mathrm{L}$ [23].

According to this test, 22 patients were found to have an impaired urinary acidification ability (Table 3 ). These patients were then analyzed by genetic testing with sequencing of 5 genes: SLC4A1, ATP6V1B1, ATP6V0A4, FOXI1, and WDR72. All patients in the study submitted an informed consent. This study was carried out according to the Declaration of Helsinki. The study is exempt from ethical committee approval since genetic testing was part of clinical practice.

\section{Gene Panel Testing}

Three genes have been selected to be analyzed by next-generation sequencing based on their previous association with dRTA, SLC4A1 (OMIM 109270), ATP6V1B1 (OMIM 192132), and ATP6V0A4 (OMIM 605239). Libraries, emulsion PCR, sample enrichment, and sequencing were performed on an Ion Torrent platform, using Life Technologies reagents (Thermo Fisher Scientific), following manufacturer recommendations. For each sample, an average coverage of $500 \mathrm{X}$ for $98 \%$ of the exons was obtained. Reads alignment, variant calling, and annotation were done using the Ion Reporter software. For most variants, a confirmation by Sanger sequencing was not deemed necessary, however the 2 variants in SLCAA1 were confirmed by Sanger analysis. Sanger sequencing was also done on a few variants to confirm they were sequencing artifacts. Variant frequency and significance were evaluated on different database: gnomAD, ClinVar, and Varsome until May 2020.

\section{Sanger Sequencing of FOXI1 and WDR72}

Recent reports highlighted a potential role in dRTA for FOXI1(OMIM 601093) and WDR72(OMIM 613214). Those genes were not included in the original gene panel and for this reason were sequenced by Sanger sequencing.

Intronic primers flanking coding exons were designed using Primer3 application on the UCSC genome browser (primer sequences will be provided upon request). Each amplicon was PCR amplified using the following standard cycles: $95^{\circ} \mathrm{C} 5 \mathrm{~min}, 95^{\circ} \mathrm{C} 30$ $\mathrm{s}, 60^{\circ} \mathrm{C} 30 \mathrm{~s}, 72^{\circ} \mathrm{C} 15 \mathrm{~s}$, for 34 cycles, and final extension at $72^{\circ} \mathrm{C}$ for $15 \mathrm{~min}$. From each amplicon, $2.5 \mu \mathrm{L}$ was purified with $0.5 \mu \mathrm{L}$ of a 1:1 mixture of exonuclease III and shrimp alkaline phosphatase at $37^{\circ} \mathrm{C}$ for $15 \mathrm{~min}$ followed by heat inactivation at $80^{\circ} \mathrm{C}$. Cleaned up PCR product was sequenced using a BigDye terminator v3.1 Cycle Sequencing Kit (Applied Biosystems, Foster City, CA) in a final volume of $10 \mu \mathrm{L}$ and run on a 3130 Genetic Analyzer (Applied Biosystems, Foster City, CA). The electropherograms were analyzed by Sequencing Analysis v5.2 software (Applied Biosystems, Foster City, CA).

\section{Results}

We identified 22 stone formers with an impaired urinary acidification ability and a positive family history of nephrolithiasis. In order to test whether idRTA is related to dRTA, we tested all 5 genes previously involved in familial and sporadic cases of RTA, SLC4A1 (OMIM 109270), ATP6V1B1 (OMIM 192132), ATP6V0A4
(OMIM 605239), FOXI1 (OMIM 601093), and WDR72 (OMIM 613214). We considered potentially "pathogenic or of uncertain clinical significance" all variants with a frequency in the gnom $A D$ database of less than $1 / 1,000$. Because 3 genes (SLC4A1, ATP6V1B1, and ATP6V0A4) have been firmly implicated in the pathogenesis of RTA, there are records of variants in ClinVar that helped to assess the variants newly discovered. We identified in 2 patients of our cohort 2 heterozygous variants in the $S L$ $C 4 A 1$ gene. This gene has been implicated both in a dominant and recessive form of dRTA. The first variant was a c. $92 \mathrm{~T}>\mathrm{G}$ predicting a p.(Met31Arg) in a 44-year-old man, recurrent stone former (mainly apatite, but also some calcium oxalate), with hypercalciuria, but normal bone mineral density. His family history revealed 1 uncle with kidney stones. The second variant was a c.1976T >C predicting a p.(Phe659Ser) in a 56-year-old woman with a history of reduced bone mineral density and severe restrictive ventilation disorder who passed 1 stone $(35 \%$ apatite, $65 \%$ calcium oxalate). She was found to have bilateral nephrocalcinosis and hypocitraturia. Her family history revealed 1 sister with kidney stones.

Variant p.(Met31Arg) has a frequency in gnomAD of $1 / 1,00,000$ because it has been reported only 3 times in the whole gnom $\mathrm{AD}$ population, two of them in the Latino population and one in "other," and never reported in the European population. To perform the functional evaluation of the aminoacidic change, we submitted to Varsome that considered this variant overall benign, given the outcome of 11 computational predictions. Moreover, this variant is located in the amino terminal portion of the protein in a region without a specific secondary structure that is not conserved across the evolution (online suppl. Table 2).

Variant p.(Phe659Ser) has never been reported in gno$\mathrm{mAD}$ and, according to several computational in silico predictions in Varsome, has only pathogenic predictions versus no benign (online suppl. Table 3 ). The phenylamine residue is highly conserved across the evolution, and even though it is not part of a well-defined secondary structure, it is located in proximity of Arg589 that is the most common pathogenic variant in autosomal dominant cases of RTA.

\section{Discussion}

To estimate the prevalence of genetic defects in idRTA, we investigated stone formers whose clinical features were suspicious of idRTA and in whom secondary causes 
of dRTA were excluded. They were analyzed by sequencing 5 genes: SLC4A1, ATP6V1B1, ATP6V0A4, FOXI1, and WDR72.

In our study, out of all the patients for whom there was a clinical suspicion of having idRTA and who had a definite family history positive for nephrolithiasis [27], only two were found to have genetic variants of SLC4A1, leading to a prevalence of genetic mutations of $10 \%$. Although we did not investigate the relatives of the 2 subjects, the clinical pattern of nephrolithiasis inheritance is consistent with an autosomal dominant segregation as expected for dRTA caused by mutations in SLC4A1. This finding extends to variants in $S L C 4 A 1$, the possibility to cause incomplete forms of dRTA. Since one of the two SLC4A1 mutations has never been reported before and the other one was found only in few cases, we hereby describe the mutations suggesting a possible pathogenetic role in idRTA.

The first variant c.92T $>$ G predicting a p.(Met31Arg) was classified according to ClinVar, functional predictions on Varsome and gnomAD frequency. This is a variant with a very low frequency in the general population $(3 / 248.470)$. It has never been reported in ClinVar, but at the same nucleotide position, there is a change in c. $92 \mathrm{~T}>\mathrm{C}$ predicting a p.(Met31T) that is considered likely benign. In Varsome, this variant has overall a benign evaluation because it is likely located in a nonconserved amino acid residue.

For the second variant c.1976T $>$ C predicting a $\mathrm{p}$. (Phe659Ser), we followed the same scheme. In ClinVar and in gnomAd, this variant has never been reported, and all functional predictions in Varsome point to a pathogenic role for this aminoacidic substitution. Moreover, this variant is in the same functional domain of the protein of the most common variant found in SLC4A1.

The low prevalence of mutations that we have observed in stone formers with idRTA is a much lower figure than overt dRTA, in which $70 \%$ of patients with a clinical diagnosis of hereditary dRTA has identified causative mutations in the currently known genes [28]. However, this study highlights that a specific role in idRTA might be played by the SLC4A1 gene, among the 5 genes known so far in dRTA. More patients with idRTA should be tested to confirm these data.

Other genetic and nongenetic causes are involved in the majority of idRTA cases observed in stone formers. Among the former causes, mutations in other genes may interfere with and reduce the function of the alpha-IC transporters. In a recent article, Merkulova et al. [29] re- ported that targeted deletion of the nuclear receptor coactivator 7 gene, encoding for a protein that interacts and is co-expressed with vacuolar-ATPases in alpha-ICs, in mice leads to a persistently high urine $\mathrm{pH}$ and hypobicarbonatemia after an $\mathrm{NH}_{4} \mathrm{Cl}$ test. Single-nucleotide polymorphisms in intron 3 of SLC4A1 have also the potential to interfere with AE1 mRNA transcription in kidney cells, therefore leading to idRTA [30]. In reference to possible nongenetic causes, although we carefully ruled out typical secondary causes of dRTA, the occurrence of other subtle secondary damages of the distal tubule might still be possible.

In conclusion, variants in the SLC4A1 gene may be responsible for cases of idRTA in renal stone formers. Although the prevalence of known monogenic forms among idRTA is relatively low and other genetic and nongenetic causes should be considered in patients affected by idRTA, it should be noted that idRTA is more frequent than dRTA and its diagnosis is more complicated. This suggests that idRTA prevalence may be higher in the general population than currently known. Moreover, it is important to note that bone metabolism also appears to be involved in idRTA: $91 \%$ of our patients who had undergone dual-energy X-ray absorptiometry had reduced bone mass, most likely due to bone buffering of chronically retained $\mathrm{H}^{+}$ions [23]. Therefore, early recognition, appropriate treatment with alkali, and prevention of complications are crucial in these patients, and genetic testing may represent a cost-effective tool to overcome practical limitations of such goals. In addition, this may allow nephrologists to provide idRTA patients with a more personalized care.

\section{Statement of Ethics}

The study complied with the Helsinki Declaration II. The study is exempt from ethical committee approval since genetic testing, acidification, and laboratory tests were part of clinical practice. All patients have given their written informed consent.

\section{Conflict of Interest Statement}

Prof Pietro Manuel Ferraro received consultant fees/grant support from Allena Pharmaceuticals, Alnylam, AstraZeneca, BioHealth Italia and Vifor Fresenius and author royalties from UpToDate; all the other authors have no potential conflicts of interest to disclose. 


\section{Funding Sources}

The authors did not receive any funding.

\section{Author Contributions}

Dr. Viola D'Ambrosio drafted the manuscript and contributed to the conception and design of the study. Dr. Alessia Azzarà, Dr. Eugenio Sangiorgi and Prof. Fiorella Gurrieri contributed to draft- ing the manuscript and data acquisition, analysis, and interpretation. Prof. Bernhard Hess, Prof. Giovanni Gambaro and Prof. Pietro Manuel Ferraro contributed to the conception and design of the study; data acquisition, analysis, and interpretation; and supervision and critical revision of the final work. Prof. Pietro Manuel Ferraro and Dr. Viola D'Ambrosio are members of the European Reference Network for Rare Kidney Diseases (ERKNet) (Project ID No. 739532).

\section{References}

1 Santos F, Gil-Peña H, Alvarez-Alvarez S. Renal tubular acidosis. Curr Opin Pediatr. 2017; 29(2):206-10.

2 Trepiccione F, Prosperi F, de la Motte LR, Hübner CA, Chambrey R, Eladari D, et al. New findings on the pathogenesis of distal renal tubular acidosis. Kidney Dis. 2017;3(3): 98-105.

3 Palazzo V, Provenzano A, Becherucci F, Sansavini G, Mazzinghi B, Orlandini V, et al. The genetic and clinical spectrum of a large cohort of patients with distal renal tubular acidosis. Kidney Int. 2017;91:1243-55.

4 Bushinsky DA, Frick KK. The effects of acid on bone. Curr Opin Nephrol Hypertens. 2000;9:369-79.

5 Rodriguez Soriano J. Renal tubular acidosis: the clinical entity. J Am Soc Nephrol. 2002;13: 2160-70.

6 Ferraro PM, D’Addessi A, Gambaro G. When to suspect a genetic disorder in a patient with renal stones, and why. Nephrol Dial Transplant. 2013;28:811-20.

7 Mohebbi N, Wagner CA. Pathophysiology, diagnosis and treatment of inherited distal renal tubular acidosis. J Nephrol. 2018;31:51122.

8 Alexander RT, Law L, Gil-Peña H, Greenbaum LA, Santos F. Hereditary distal renal tubular acidosis. GeneReviews ${ }^{\circ}$ [Internet]. Seattle (WA): University of Washington; 1993 2020, 2019 Oct 10.

9 Vallés PG, Batlle D. Hypokalemic distal renal tubular acidosis. Adv Chronic Kidney Dis. 2018;25(4):303-20.

10 Bertocchio JP, Genetet S, Da Costa L, Walsh SB, Knebelmann B, Galimand J, et al. Red blood cell AE1/Band 3 transports in dominant distal renal tubular acidosis patients. Kidney Int Rep. 2020;5:348-57.

11 Rysava R, Tesar V, Jirsa M Jr, Brabec V, Jarolím P. Incomplete distal renal tubular acidosis coinherited with a mutation in the band 3 (AE1) gene. Nephrol Dial Transplant. 1997; 12:1869-73.
12 Trepiccione F, Gerber SD, Grahammer F, López-Cayuqueo KI, Baudrie V, Păunescu TG, et al. Renal Atp6ap2/(Pro)renin receptor is required for normal vacuolar $\mathrm{H}+$-ATPase function but not for the renin-angiotensin system. J Am Soc Nephrol. 2016;27:3320-30.

13 Wrong BYO, Davies HEF. The excretion of acid in renal disease. Q J Med. 1959;28:259313.

14 Bobulescu IA, Maalouf NM, Capolongo G, Adams-Huet B, Rosenthal TR, Moe OW, et al. Renal ammonium excretion after an acute acid load: blunted response in uric acid stone formers but not in patients with type 2 diabetes. Am J Physiol Renal Physiol. 2013 Nov; 305(10):F1498-503.

15 Walsh SB, Shirley DG, Wrong OM, Unwin RJ. Urinary acidification assessed by simultaneous furosemide and fludrocortisone treatment: an alternative to ammonium chloride. Kidney Int. 2007;71:1310-6.

16 Shavit L, Chen L, Ahmend F, Ferraro PM, Moochhala S, Walsh SB, et al. Selective screening for distal renal tubular acidosis in recurrent kidney stone formers: initial experience and comparison of the simultaneous furosemide and fludrocortisone test with the short ammonium chloride test. Nephrol Dial Transplant. 2016;31:1870-6.

17 Goldfarb DS. Refining diagnostic approaches in nephrolithiasis: incomplete distal renal tubular acidosis. Clin J Am Soc Nephrol. 2017; 12(9):1380-2

18 Dhayat NA, Gradwell MW, Pathare G, Anderegg M, Schneider L, Luethi D, et al. Furosemide/fludrocortisone test and clinical parameters to diagnose incomplete distal renal tubular acidosis in kidney stone formers. Clin J Am Soc Nephrol. 2017;12:1507-17.

19 Zhang J, Fuster DG, Cameron MA, Quiñones $\mathrm{H}$, Griffith C, Xie X-S, et al. Incomplete distal renal tubular acidosis from a heterozygous mutation of the V-ATPase B1 subunit. Am J Physiol Renal Physiol. 2014;307:F1063-71.
20 Dhayat NA, Schaller A, Albano G, Poindexter J, Griffith C, Pasch A, et al. The vacuolar H+ATPase B1 subunit polymorphism p.E161K associates with impaired urinary acidification in recurrent stone formers. J Am Soc Nephrol. 2016;27:1544-54.

21 Imai E, Kaneko S, Mori T, Okado T, Uchida $\mathrm{S}$, Tsukamoto Y. A novel heterozygous mutation in the ATP6V0A4 gene encoding the VATPase a4 subunit in an adult patient with incomplete distal renal tubular acidosis. Clin kidney J. 2016;9:424-8.

22 Weger M, Deutschmann H, Weger W, Kotanko P, Skrabal F. Incomplete renal tubular acidosis in "primary"osteoporosis. Osteoporos Int. 1999;10:325-9.

23 Sromicki JJ, Hess B. Abnormal distal renal tubular acidification in patients with low bone mass: prevalence and impact of alkali treatment. Urolithiasis. 2017;45:263-9.

24 Arampatzis S, Röpke-Rieben B, Lippuner K, Hess B. Prevalence and densitometric characteristics of incomplete distal renal tubular acidosis in men with recurrent calcium nephrolithiasis. Urol Res. 2012;40:53-9.

25 Alonso-Varela M, Gil-Peña H, Santos F. Incomplete distal renal tubular acidosis in children. Acta Paediatr. 2020;109:2243-50.

26 Hess B. Acid-base metabolism: implications for kidney stones formation. Urol Res. 2006; 34:134-8.

27 Fuster DG, Moe OW. Incomplete distal renal tubular acidosis and kidney stones. Adv Chronic Kidney Dis. 2018;25(4):366-74.

28 Enerbäck S, Nilsson D, Edwards N, Heglind M, Alkanderi S, Ashton E, et al. Acidosis and deafness in patients with recessive mutations in FOXI1. J Am Soc Nephrol. 2018;29(3): 1041-8.

29 Merkulova M, Păunescu TG, Nair AV, Wang CY, Capen DE, Oliver PL, et al. Targeted deletion of the Ncoa7 gene results in incomplete distal renal tubular acidosis in mice. Am J Physiol Renal Physiol. 2018;315:F173-85.

30 Takeuchi T, Hattori-Kato M, Okuno Y, Kanatani A, Zaitsu M, Mikami K. A single nucleotide polymorphism in kidney anion exchanger 1 gene is associated with incomplete type 1 renal tubular acidosis. Sci Rep. 2016;6:35841. 